\title{
Maximum Margin Multi-Dimensional Classification
}

\author{
Bin-Bin Jia, ${ }^{1,2,3}$ Min-Ling Zhang ${ }^{1,3,4 *}$ \\ ${ }^{1}$ School of Computer Science and Engineering, Southeast University, Nanjing 210096, China \\ ${ }^{2}$ College of Electrical and Information Engineering, Lanzhou University of Technology, Lanzhou 730050, China \\ ${ }^{3}$ Key Laboratory of Computer Network and Information Integration (Southeast University), Ministry of Education, China \\ ${ }^{4}$ Collaborative Innovation Center of Wireless Communications Technology, China \\ $\{$ jiabb, zhangml\}@seu.edu.cn
}

\begin{abstract}
Multi-dimensional classification (MDC) assumes heterogenous class spaces for each example, where class variables from different class spaces characterize semantics of the example along different dimensions. Due to the heterogeneity of class spaces, the major difficulty in designing marginbased MDC techniques lies in that the modeling outputs from different class spaces are not comparable to each other. In this paper, a first attempt towards maximum margin multidimensional classification is investigated. Following the onevs-one decomposition within each class space, the resulting models are optimized by leveraging classification margin maximization on individual class variable and model relationship regularization across class variables. We derive convex formulation for the maximum margin MDC problem, which can be tackled with alternating optimization admitting QP or closed-form solution in either alternating step. Experimental studies over real-world MDC data sets clearly validate effectiveness of the proposed maximum margin MDC techniques.
\end{abstract}

\section{Introduction}

In multi-dimensional classification, each training example is represented by a single instance while associated with multiple class variables (Read, Bielza, and Larrañaga 2014; Ma and Chen 2018; Jia and Zhang 2019). Here, each class variable corresponds to one specific class space which characterizes the semantics of an object along one dimension. Many real-world problems can be naturally formalized under MDC frameworks (Rodríguez et al. 2012; Borchani et al. 2013; Sagarna et al. 2014; Serafino et al. 2015). For example, a news document can be characterized from the topic dimension (with possible classes sports, politics, social, Sci\&Tech, etc.), from the mood dimension (with possible classes good news, neutral news, bad news), and from the zone dimension (with possible classes domestic, intra/inter-continental, etc.).

Formally speaking, let $\mathcal{X}=\mathbb{R}^{d}$ be the $d$-dimensional input (feature) space and $\mathcal{Y}=C_{1} \times C_{2} \times \cdots \times C_{q}$ be the output space which corresponds to the Cartesian product of $q$ class

\footnotetext{
${ }^{*}$ Corresponding author Copyright (C) 2020, Association for the Advancement of Artificial Intelligence (www.aaai.org). All rights reserved.
}

spaces. Here, each class space $C_{j}(1 \leq j \leq q)$ consists of $K_{j}$ possible class labels, i.e., $C_{j}=\left\{c_{1}^{j}, c_{2}^{j}, \ldots, c_{K_{j}}^{j}\right\}$, among which only one is relevant to the example. Furthermore, let $\mathcal{D}=\left\{\left(\boldsymbol{x}_{i}, \boldsymbol{y}_{i}\right) \mid 1 \leq i \leq N\right\}$ be the MDC training set with $N$ training examples, where $\boldsymbol{x}_{i} \in \mathcal{X}$ is a $d$ dimensional feature vector and $\boldsymbol{y}_{i}=\left[y_{i 1}, y_{i 2}, \ldots, y_{i q}\right]^{\top} \in$ $\mathcal{Y}$ is the associated class vector, each of which is one possible value in the corresponding class space, i.e., $y_{i j} \in C_{j}$. Then, the task of multi-dimensional classification is to induce a predictive function $f: \mathcal{X} \mapsto \mathcal{Y}$ from $\mathcal{D}$ which can assign a proper class vector $f(\boldsymbol{x}) \in \mathcal{Y}$ for the unseen instance $\boldsymbol{x}$.

To accomplish the task of learning from MDC examples, the most intuitive strategy is to induce a number of independent multi-class classifiers, one per class space. However, this strategy completely ignores potential dependencies among class variables which would impact the generalization performance of induced predictive model. Therefore, most existing approaches try to model class dependencies in different ways, such as specifying chaining order over class variables (Zaragoza et al. 2011; Read, Martino, and Luengo 2014), assuming directed acyclic graph (DAG) structure over class variables (Bielza, Li, and Larrañaga 2011; Batal, Hong, and Hauskrecht 2013; Zhu, Liu, and Jiang 2016; Bolt and van der Gaag 2017; Gil-Begue, Larrañaga, and Bielza 2018; Benjumeda, Bielza, and Larrañaga 2018), and partitioning class variables into groups (Read, Bielza, and Larrañaga 2014), etc.

To derive margin-based techniques for multi-dimensional classification, the major difficulty lies in that the modeling outputs from different class spaces are not directly comparable. In this paper, we make a first attempt to adapt maximum margin technique for multi-dimensional classification, and propose a novel approach named $\mathrm{M}^{3} \mathrm{MDC}$, i.e., MaxiMum Margin for Multi-Dimensional Classification. Specifically, based on one-vs-one decomposition within each class space, the multi-dimensional classification models are optimized by maximizing classification margin on individual class variable and regularizing model relationship across class variables. The resulting convex formulation is solved with alternating optimization admitting QP or closed-form solution in either alternating step. Comparative studies against 
other well-established MDC approaches clearly validate the effectiveness of the proposed approach.

The rest of this paper is organized as follows. Firstly, technical details of the proposed approach are introduced. Secondly, related works on MDC are briefly discussed. Thirdly, experimental results of comparative studies are reported. Finally, we conclude this paper.

\section{The Maximum Margin MDC Approach}

The common premise of margin-based approaches is that different modeling outputs are comparable. However, due to MDC's inherent property that each class variable corresponds to one heterogenous class space, the modeling outputs from different class spaces are not directly comparable. In this section, we present technical details of the $\mathrm{M}^{3} \mathrm{MDC}$ approach which considers the margins between each pair of class labels in the same class space.

Following the same notations given in previous section, it is easy to know that there are totally $m=\sum_{j=1}^{q} \frac{K_{j}\left(K_{j}-1\right)}{2}$ pairs of class labels across all class spaces. To obtain margins between each pair of class labels, one-vs-one $(\mathrm{OvO})$ decomposition is made accordingly. Without loss of generality, for the $i$ th pair of class labels $l_{+}^{i}$ and $l_{-}^{i}$, let $\mathcal{D}^{i}=$ $\left\{\left(\boldsymbol{x}_{j}^{i}, y_{j}^{i}\right) \mid 1 \leq j \leq n_{i}\right\}$ be the corresponding OvO decomposition training set. Here, $\boldsymbol{x}_{j}^{i} \in \mathcal{X}, y_{j}^{i}$ equals +1 when $l_{+}^{i}$ is relevant and -1 when $l_{-}^{i}$ is relevant, $n_{i}$ is the number of training examples in $\mathcal{D}$ for which either $l_{+}^{i}$ or $l_{-}^{i}$ is relevant. Assuming that hyperplane $\left(\boldsymbol{w}_{i}, b_{i}\right)$ can perfectly classify examples in $\mathcal{D}^{i}$, the margin of $\left(\boldsymbol{w}_{i}, b_{i}\right)$ can be defined as $2 /\left\|\boldsymbol{w}_{i}\right\|$ by appropriately normalizing $\left(\boldsymbol{w}_{i}, b_{i}\right)$ (Cortes and Vapnik 1995), where $\|\cdot\|$ denotes the vector norm. We can get the maximum margin hyperplane by maximizing $2 /\left\|\boldsymbol{w}_{i}\right\|$ which is equivalent to minimizing $\left\|\boldsymbol{w}_{i}\right\|^{2} / 2$. Considering all pairs of class labels, let $\mathbf{W}=\left[\boldsymbol{w}_{1}, \ldots, \boldsymbol{w}_{m}\right] \in \mathbb{R}^{d \times m}$ and $\boldsymbol{b}=\left(b_{1}, \ldots, b_{m}\right)^{\top}$, and for a more general case that training examples in each $\mathcal{D}^{i}$ can't be separated perfectly, slack variables $\boldsymbol{\xi}=\left(\xi_{1}^{1}, \ldots, \xi_{n_{1}}^{1}, \ldots, \xi_{1}^{m}, \ldots, \xi_{n_{m}}^{m}\right)^{\top}$ can be introduced to model the empirical risk. Then, we can get the following maximum margin formulation for MDC:

$$
\begin{aligned}
\min _{\mathbf{W}, \boldsymbol{b}, \boldsymbol{\xi}} & \sum_{i=1}^{m} \sum_{j=1}^{n_{i}} \xi_{j}^{i}+\frac{\lambda_{1}}{2} \operatorname{tr}\left(\mathbf{W} \mathbf{W}^{\top}\right) \\
\text { s.t. } & y_{j}^{i}\left(\left\langle\boldsymbol{w}_{i}, \boldsymbol{x}_{j}^{i}\right\rangle+b_{i}\right) \geq 1-\xi_{j}^{i}, \\
& \xi_{j}^{i} \geq 0, i=1, \ldots, m, j=1, \ldots, n_{i}
\end{aligned}
$$

where $\langle\cdot, \cdot\rangle$ denotes inner product of two vectors, $\operatorname{tr}(\cdot)$ denotes the trace of a square matrix, and $\lambda_{1}$ is a regularization parameter. The formulation in Eq.(1) just independently deals with each pair of class labels, i.e., dependencies among class spaces are ignored. Following the idea in (Zhang and Yeung 2014; Liu et al. 2016; Ma and Chen 2019), we model the relationships among all $\boldsymbol{w}_{i} \mathrm{~s}$ in $\mathbf{W}$ with the column covariance matrix of $\mathbf{W}$. Thereafter, the above optimization problem turns out to be:

$$
\begin{aligned}
& \min _{\mathbf{W}, \boldsymbol{b}, \boldsymbol{\xi}, \mathbf{C}} \sum_{i=1}^{m} \sum_{j=1}^{n_{i}} \xi_{j}^{i}+\frac{\lambda_{1}}{2} \operatorname{tr}\left(\mathbf{W} \mathbf{W}^{\top}\right) \\
&+\frac{\lambda_{2}}{2} \operatorname{tr}\left(\mathbf{W} \mathbf{C}^{-1} \mathbf{W}^{\top}\right) \\
& \text { s.t. } \quad \mathbf{C} \succeq 0, \operatorname{tr}(\mathbf{C}) \leq 1, \\
& y_{j}^{i}\left(\left\langle\boldsymbol{w}_{i}, \boldsymbol{x}_{j}^{i}\right\rangle+b_{i}\right)>1-\xi_{j}^{i}, \\
& \xi_{j}^{i} \geq 0, i=1, \ldots, m, j=1, \ldots, n_{i}
\end{aligned}
$$

Here $\mathbf{C} \succeq 0$ means that $\mathbf{C}$ is positive semi-definite which corresponds to a covariance matrix, and $\operatorname{tr}(\mathbf{C}) \leq 1$ is used to penalize its complexity. $\lambda_{2}$ is another regularization parameter.

Obviously, the first two terms in objective function are convex with respect to $\mathbf{W}$ and $\boldsymbol{b}$, and it has been proved in (Zhang and Yeung 2014) that the third term in the objective function is also a convex function with respect to $\mathbf{W}, \boldsymbol{b}$ and C. So the optimization problem in Eq.(2) is jointly convex.

However, it is not easy to solve this optimization problem directly because of the non-linear and non-smooth constraint $\mathbf{C} \succeq 0$. Here, we use an alternating method to solve it efficiently. Specifically, the objective function with respect to $\mathbf{W}$ and $\boldsymbol{b}$ is firstly optimized when $\mathbf{C}$ is fixed, and then it is optimized with respect to $\mathbf{C}$ when $\mathbf{W}$ and $\boldsymbol{b}$ are fixed. These two steps are repeated until convergence. Technical details of the two alternating steps are introduced as follows.

Optimizing with respect to $\mathrm{W}$ and $b$ when $\mathrm{C}$ is fixed. When $\mathbf{C}$ is fixed, we can reformulate the optimization problem in Eq.(2) as follows:

$$
\begin{aligned}
\min _{\mathbf{W}, \boldsymbol{b}, \boldsymbol{\xi}} & \sum_{i=1}^{m} \sum_{j=1}^{n_{i}} \xi_{j}^{i}+\frac{\lambda_{1}}{2} \operatorname{tr}\left(\mathbf{W} \mathbf{W}^{\top}\right) \\
& +\frac{\lambda_{2}}{2} \operatorname{tr}\left(\mathbf{W} \mathbf{C}^{-1} \mathbf{W}^{\top}\right) \\
\text { s.t. } & y_{j}^{i}\left(\left\langle\boldsymbol{w}_{i}, \boldsymbol{x}_{j}^{i}\right\rangle+b_{i}\right)>1-\xi_{j}^{i}, \\
& \xi_{j}^{i} \geq 0, i=1, \ldots, m, j=1, \ldots, n_{i}
\end{aligned}
$$

The Lagrangian of the above problem is given by:

$$
\begin{aligned}
\mathcal{L}(\mathbf{W}, \boldsymbol{b}, \boldsymbol{\xi}, \boldsymbol{\alpha}, \boldsymbol{\beta})=\sum_{i=1}^{m} \sum_{j=1}^{n_{i}} \xi_{j}^{i}+\frac{\lambda_{1}}{2} \operatorname{tr}\left(\mathbf{W} \mathbf{W}^{\top}\right) \\
+\frac{\lambda_{2}}{2} \operatorname{tr}\left(\mathbf{W} \mathbf{C}^{-1} \mathbf{W}^{\top}\right) \\
-\sum_{i=1}^{m} \sum_{j=1}^{n_{i}} \alpha_{j}^{i}\left[y_{j}^{i}\left(\left\langle\boldsymbol{w}_{i}, \boldsymbol{x}_{j}^{i}\right\rangle+b_{i}\right)-1+\xi_{j}^{i}\right] \\
-\sum_{i=1}^{m} \sum_{j=1}^{n_{i}} \beta_{j}^{i} \xi_{j}^{i}
\end{aligned}
$$

where $\alpha_{j}^{i}, \beta_{j}^{i} \geq 0$. Then, the gradients of $\mathcal{L}$ are calculated with respected to $\mathbf{W}, b_{i}$ and $\xi_{j}^{i}$, and we can obtain the fol- 
lowing equations by setting them to be 0 :

$$
\begin{aligned}
\frac{\partial \mathcal{L}}{\partial \mathbf{W}}=0 & \Rightarrow \\
\mathbf{W} & =\sum_{i=1}^{m} \sum_{j=1}^{n_{i}} \alpha_{j}^{i} y_{j}^{i} \boldsymbol{x}_{j}^{i} \boldsymbol{e}_{i}^{\top} \mathbf{C}\left(\lambda_{1} \mathbf{I}_{m}+\lambda_{2} \mathbf{C}\right)^{-1} \\
\frac{\partial \mathcal{L}}{\partial b_{i}}=0 & \Rightarrow \sum_{j=1}^{n_{i}} \alpha_{j}^{i} y_{j}^{i}=0,(1 \leq i \leq m) \\
\frac{\partial \mathcal{L}}{\partial \xi_{j}^{i}}=0 & \Rightarrow \alpha_{j}^{i}+\beta_{j}^{i}=1
\end{aligned}
$$

where $\boldsymbol{e}_{i}$ is the $i$ th column vector of identity matrix $\mathbf{I}_{m}$. Plugging Eq.(5) Eq.(7) into Eq.(4), the dual problem, i.e., $\max _{\boldsymbol{\alpha}} \min _{\boldsymbol{W}, \boldsymbol{b}} \mathcal{L}(\mathbf{W}, \mathbf{b})$, can be equivalently formulated as:

$$
\begin{aligned}
\min _{\boldsymbol{\alpha}} & \frac{1}{2} \sum_{i_{1}=1}^{m} \sum_{j_{1}=1}^{n_{i_{1}}} \sum_{i_{2}=1}^{m} \sum_{j_{2}=1}^{n_{i_{2}}} \alpha_{j_{1}}^{i_{1}} \alpha_{j_{2}}^{i_{2}} y_{j_{1}}^{i_{1}} y_{j_{2}}^{i_{2}} M_{i_{1} i_{2}}\left\langle\boldsymbol{x}_{j_{1}}^{i_{1}}, \boldsymbol{x}_{j_{2}}^{i_{2}}\right\rangle \\
& -\sum_{i=1}^{m} \sum_{j=1}^{n_{i}} \alpha_{j}^{i} \\
& \text { s.t. } \sum_{j=1}^{n_{i}} \alpha_{j}^{i} y_{j}^{i}=0(1 \leq i \leq m), 0 \leq \alpha_{j}^{i} \leq 1
\end{aligned}
$$

where $\mathbf{M}=\left(\lambda_{1} \mathbf{I}_{m}+\lambda_{2} \mathbf{C}\right)^{-\top} \mathbf{C}^{\top}$, and $M_{i_{1} i_{2}}$ denotes the element in $i_{1}$ th row and $i_{2}$ th column of M. $\boldsymbol{\alpha}=$ $\left(\alpha_{1}^{1}, \ldots, \alpha_{n_{1}}^{1}, \ldots, \alpha_{1}^{m}, \ldots, \alpha_{n_{m}}^{m}\right)^{\top} \in \mathbb{R}^{\sum_{j=1}^{m} n_{j} \times 1}$. Eq.(8) is a standard quadratic programming $(\mathrm{QP})$ problem with $m$ equality constraints, but the number of $\alpha_{j}^{i}$ s, i.e., $\sum_{j=1}^{m} n_{j}$, is usually too large making this QP problem difficult to be solved directly. Here, we decompose it into $m$ sub-QP problems with only one equality constraint as follows:

$$
\begin{aligned}
& \min _{\boldsymbol{\alpha}^{i}} \frac{1}{2} \sum_{j_{1}=1}^{n_{i}} \sum_{j_{2}=1}^{n_{i}} \alpha_{j_{1}}^{i} \alpha_{j_{2}}^{i} y_{j_{1}}^{i} y_{j_{2}}^{i} M_{i i}\left\langle\boldsymbol{x}_{j_{1}}^{i}, \boldsymbol{x}_{j_{2}}^{i}\right\rangle \\
&-\sum_{j=1}^{n_{i}}\left(1-S_{j}^{i}\right) \alpha_{j}^{i} \\
& \text { s.t. } \quad \sum_{j=1}^{n_{i}} \alpha_{j}^{i} y_{j}^{i}=0,0 \leq \alpha_{j}^{i} \leq 1
\end{aligned}
$$

where $1 \leq i \leq m, S_{j}^{i}=y_{j}^{i} \sum_{i_{1} \neq i} \frac{1}{2}\left(M_{i i_{1}}+\right.$ $\left.M_{i_{1} i}\right) \sum_{j_{1}=1}^{n_{i_{1}}} \alpha_{j_{1}}^{i_{1}} y_{j_{1}}^{i_{1}}\left\langle\boldsymbol{x}_{j}^{i}, \boldsymbol{x}_{j_{1}}^{i_{1}}\right\rangle$, and $\boldsymbol{\alpha}^{i}=\left(\alpha_{1}^{i}, \ldots, \alpha_{n_{i}}^{i}\right)^{\top} \in$ $\mathbb{R}^{n_{i} \times 1}$. To solve the problem in Eq.(8), we can initialize $\boldsymbol{\alpha}=\mathbf{0}$, and then repeatedly solve the $m$ sub-QP problems in Eq. (9) until all $\alpha_{j}^{i}$ s meet Karush-Kuhn-Tucker (KKT) conditions.

Here, the values of $\mathbf{W}$ and $\boldsymbol{b}$ need to be obtained for validating KKT conditions. For $\mathbf{W}$, it just needs to plug $\boldsymbol{\alpha}$ into Eq.(5). But for $\boldsymbol{b}$, the situation is somewhat complicated. When there are $\alpha_{j}^{i}$ s in $(0,1)$, we have $y_{j}^{i}\left(\left\langle\boldsymbol{w}_{i}, \boldsymbol{x}_{j}^{i}\right\rangle+b_{i}\right)=1$, it's easy to know that $b_{i}=y_{j}^{i}-\left\langle\boldsymbol{w}_{i}, \boldsymbol{x}_{j}^{i}\right\rangle$. However, when there aren't $\alpha_{j}^{i} \mathrm{~s}$ in $(0,1)$, i.e., either $\alpha_{j}^{i}=0$ or $\alpha_{j}^{i}=1$, we need to solve the inequalities. In this case, when $\alpha_{j}^{i}=0, b_{i}$ should meet $y_{j}^{i}\left(\left\langle\boldsymbol{w}_{i}, \boldsymbol{x}_{j}^{i}\right\rangle+b_{i}\right) \geq 1$, while when $\alpha_{j}^{i}=1, b_{i}$ should meet $y_{j}^{i}\left(\left\langle\boldsymbol{w}_{i}, \boldsymbol{x}_{j}^{i}\right\rangle+b_{i}\right) \leq 1$. Then we can get many upper and lower limits of $b_{i}$, in which we select the moderate one for $b_{i}$.

Optimizing with respect to $\mathrm{C}$ when $\mathrm{W}$ and $\boldsymbol{b}$ are fixed. When $\mathbf{W}$ and $\boldsymbol{b}$ are fixed, the optimization problem in Eq.(2) for finding $\mathbf{C}$ becomes:

$$
\min _{\mathbf{C}} \operatorname{tr}\left(\mathbf{W} \mathbf{C}^{-1} \mathbf{W}^{\top}\right) \text {, s.t. } \mathbf{C} \succeq 0, \operatorname{tr}(\mathbf{C}) \leq 1
$$

As per the property of $\operatorname{tr}(\mathbf{X Y Z})=\operatorname{tr}(\mathbf{Y Z X})$ and the constraint $\operatorname{tr}(\mathbf{C}) \leq 1$, we can lower-bound the objective in Eq.(10) as:

$$
\begin{aligned}
\operatorname{tr}\left(\mathbf{W} \mathbf{C}^{-1} \mathbf{W}^{\top}\right) & =\operatorname{tr}\left(\mathbf{C}^{-1} \mathbf{W}^{\top} \mathbf{W}\right) \\
& \geq \operatorname{tr}\left(\mathbf{C}^{-1} \mathbf{W}^{\top} \mathbf{W}\right) \operatorname{tr}(\mathbf{C}) \\
& =\operatorname{tr}\left(\mathbf{C}^{-\frac{1}{2}} \mathbf{A}^{\frac{1}{2}} \mathbf{A}^{\frac{1}{2}} \mathbf{C}^{-\frac{1}{2}}\right) \operatorname{tr}\left(\mathbf{C}^{\frac{1}{2}} \mathbf{C}^{\frac{1}{2}}\right) \\
& \geq\left(\operatorname{tr}\left(\mathbf{C}^{-\frac{1}{2}} \mathbf{A}^{\frac{1}{2}} \mathbf{C}^{\frac{1}{2}}\right)\right)^{2}=\left(\operatorname{tr}\left(\mathbf{A}^{\frac{1}{2}}\right)\right)^{2}
\end{aligned}
$$

where $\mathbf{A}=\mathbf{W}^{\top} \mathbf{W}=\sum_{i_{1}=1}^{m} \sum_{j_{1}=1}^{n_{i_{1}}} \sum_{i_{2}=1}^{m} \sum_{j_{2}=1}^{n_{i_{2}}} \alpha_{j_{1}}^{i_{1}}$ $\alpha_{j_{2}}^{i_{2}} y_{j_{1}}^{i_{1}} y_{j_{2}}^{i_{2}} \mathbf{M} \boldsymbol{e}_{i_{1}} \boldsymbol{e}_{i_{2}}^{\top} \mathbf{M}^{\top}\left\langle\boldsymbol{x}_{j_{1}}^{i_{1}}, \boldsymbol{x}_{j_{2}}^{i_{2}}\right\rangle$. The last inequality in Eq.(11) holds based on the property that both $\mathbf{A}$ and $\mathbf{C}$ are symmetric as well as the following Lemma:

Lemma 1. Given $\mathbf{U}, \mathbf{V} \in \mathbb{R}^{\ell_{1} \times \ell_{2}}$, then $\operatorname{tr}\left(\mathbf{U}^{\top} \mathbf{U}\right) \operatorname{tr}\left(\mathbf{V}^{\top} \mathbf{V}\right) \geq\left(\operatorname{tr}\left(\mathbf{U}^{\top} \mathbf{V}\right)\right)^{2}$ holds. The minimum can be got when $\overline{\mathbf{U}}=\mu \cdot \mathbf{V}$ where $\mu$ is a constant.

Proof. It is easy to know,

$$
\begin{aligned}
& \operatorname{tr}\left(\mathbf{U}^{\top} \mathbf{U}\right)=\sum_{i=1}^{\ell_{2}} \sum_{j=1}^{\ell_{1}} U_{i j}^{2}=\langle\operatorname{vec} \mathbf{U}, \operatorname{vec} \mathbf{U}\rangle=\|\operatorname{vec} \mathbf{U}\|^{2} \\
& \operatorname{tr}\left(\mathbf{V}^{\top} \mathbf{V}\right)=\sum_{i=1}^{\ell_{2}} \sum_{j=1}^{\ell_{1}} V_{i j}^{2}=\langle\operatorname{vec} \mathbf{V}, \operatorname{vec} \mathbf{V}\rangle=\|\operatorname{vec} \mathbf{V}\|^{2} \\
& \operatorname{tr}\left(\mathbf{U}^{\top} \mathbf{V}\right)=\sum_{i=1}^{\ell_{2}} \sum_{j=1}^{\ell_{1}} U_{i j} V_{i j}=\langle\operatorname{vec} \mathbf{U}, \operatorname{vec} \mathbf{V}\rangle
\end{aligned}
$$

Here, vec $\mathbf{U}$, vec $\mathbf{V}$ denote the results of vectorization for $\mathbf{U}, \mathbf{V}$. As per the property of inner product $\|v e c \mathbf{U}\|$. $\|v e c \mathbf{V}\| \geq|\langle\operatorname{vec} \mathbf{U}, \operatorname{vec} \mathbf{V}\rangle|$, and take the square over both sides of this inequality, then we have $\|v e c \mathbf{U}\|^{2}$. $\|$ vec $\mathbf{V} \|^{2} \geq(\langle\operatorname{vec} \mathbf{U}, \operatorname{vec} \mathbf{V}\rangle)^{2}$. The equality relationship holds only when $\operatorname{vec} \mathbf{U}=\mu \cdot \operatorname{vec} \mathbf{V}$, i.e., $\mathbf{U}=\mu \cdot \mathbf{V}$.

According to Eq.(11), $\operatorname{tr}\left(\mathbf{W C}^{-1} \mathbf{W}^{\top}\right)$ attains its minimum value $\left(\operatorname{tr}\left(\mathbf{A}^{\frac{1}{2}}\right)\right)^{2}$ when $\operatorname{tr}(\mathbf{C})=1$ and $\mathbf{A}^{\frac{1}{2}} \mathbf{C}^{-\frac{1}{2}}=$ $\mu \mathbf{C}^{\frac{1}{2}}$ for some constant $\mu$. Therefore, the closed-form solution of $\mathbf{C}$ can be obtained (Zhang and Yeung 2014) as follows:

$$
\mathbf{C}=\frac{\left(\mathbf{W}^{\top} \mathbf{W}\right)^{\frac{1}{2}}}{\operatorname{tr}\left(\left(\mathbf{W}^{\top} \mathbf{W}\right)^{\frac{1}{2}}\right)}
$$

As the above two alternating optimizing steps converge, we can get the optimal values of $\mathbf{W}, \boldsymbol{b}$ and $\mathbf{C}$. Then, predictions 
Table 1: The pseudo-code of $\mathrm{M}^{3} \mathrm{MDC}$.

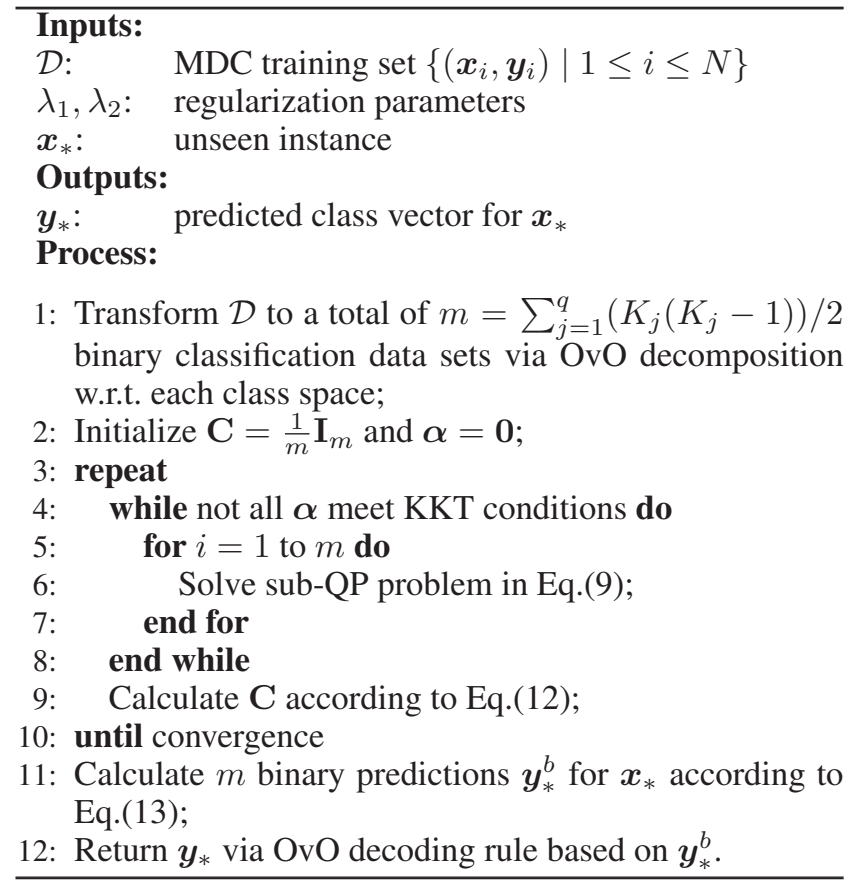

for unseen instances can be made accordingly. Specifically, for test instance $\boldsymbol{x}_{*}$, we can get its binary prediction vector $\boldsymbol{y}_{*}^{b}$ with a total of $m$ elements as follows:

$$
\begin{array}{r}
\boldsymbol{y}_{*}^{b}=\operatorname{sign}\left(\mathbf{W}^{\top} \boldsymbol{x}_{*}+\boldsymbol{b}\right) \\
=\operatorname{sign}\left(\sum_{i=1}^{m} \sum_{j=1}^{n_{i}} \alpha_{j}^{i} y_{j}^{i} \mathbf{M} \boldsymbol{e}_{i}\left\langle\boldsymbol{x}_{j}^{i}, \boldsymbol{x}_{*}\right\rangle+\boldsymbol{b}\right)
\end{array}
$$

where $\operatorname{sign}(\cdot)$ is the (element-wise) signed function. The first $\frac{K_{1}\left(K_{1}-1\right)}{2}$ elements in $\boldsymbol{y}_{*}^{b}$ belong to the first class space, the $\frac{K_{1}\left(K_{1}-1\right)}{2}+1 \sim \frac{K_{2}\left(K_{2}-1\right)}{2}$ elements belong to the second class space, and so on. Finally, we can make prediction of each class space for $\boldsymbol{x}_{*}$ via OvO decoding rule based on these binary predictions.

In summary, Table 1 presents the complete procedure of the proposed $\mathrm{M}^{3} \mathrm{MDC}$ approach. Firstly, we employ OvO decomposition for the original MDC problem per class space (Step 1). After that, an alternating optimizing process is used to solve the problem in Eq.(2) (Steps 2-10). Finally, the class vector for unseen instance is predicted based on its $m$ binary predictions (Steps 11-12).

Computational complexity. Let $\mathcal{F}_{Q P}(r)$ denote the time complexity to solve Eq.(9) with $r$ variables, and $\mathcal{F}_{S R}(s)$ denote the time complexity to solve matrix square root operation in Eq.(12) with $s \times s$ elements. ${ }^{1} \mathrm{M}^{3} \mathrm{MDC}$ has computational complexity $\mathcal{O}\left(T_{1} \cdot T_{2} \cdot m \cdot \mathcal{F}_{Q P}(N)+T_{1} \cdot \mathcal{F}_{S R}(m)\right)$ for training phase, where $T_{1}$ denotes the number of iterations

\footnotetext{
${ }^{1}$ MOSEK optimization software (https://www.mosek.com/) is used to solve Eq.(9), and built-in function sqrtm in Matlab is used to solve Eq.(12).
}

of the whole alternating optimizing process and $T_{2}$ denotes the number of iterations of $m$ sub-QP problems in Eq.(9). Moreover, $\mathcal{F}_{Q P}(N)$ is actually the maximum complexity of each Eq.(9) because the number of examples in each OvO decomposition is always less than $N$.

\section{Related Work}

Intuitively, MDC corresponds to a set of traditional multiclass classification (MCC), one per class space. However, it is better to solve the set of MCC together rather than one by one independently, because dependencies among class variables usually exist due to the fact that all these MCC problems share the same input space. Therefore, most existing MDC approaches try to model class dependencies in different ways, such as capturing pairwise interactions between class variables (Arias et al. 2016), specifying chaining order over class variables (Zaragoza et al. 2011; Read, Martino, and Luengo 2014), assuming directed acyclic graph (DAG) structure over class variables (Bielza, Li, and Larrañaga 2011; Batal, Hong, and Hauskrecht 2013; Zhu, Liu, and Jiang 2016; Bolt and van der Gaag 2017; Gil-Begue, Larrañaga, and Bielza 2018; Benjumeda, Bielza, and Larrañaga 2018), and partitioning class variables into groups (Read, Bielza, and Larrañaga 2014), etc.

Furthermore, MDC can also be regarded as a generalized version of multi-label classification (MLC) (Zhang and Zhou 2014; Gibaja and Ventura 2015) by not restricting binary-valued class variable in each class space. However, the key difference between MDC and MLC is whether the class space is heterogenous or homogeneous. Generally, MDC assumes heterogenous class spaces which characterize objects' semantics along different dimensions, while MLC assumes homogeneous class space which characterizes the relevancy of specific concepts along one dimension. In other words, the relationship between a pair of class labels from different class spaces in MDC is different from the relationship between a pair of class labels in MLC. Therefore, it is unreasonable and will get suboptimal solutions to directly align class labels from different class spaces when trying to design MDC approaches.

Maximum margin techniques have been widely used to solve MCC and MLC problems. For MCC with single-label assignment, one can derive margin-based classification models by transforming the MCC problem into a number of binary classification problems via one-vs-one, one-vs-all, and many-vs-many decomposition, or directly maximizing multi-class margins. For MLC with multi-label assignment, one can also derive margin-based classification models via binary decomposition, or by maximizing margins between relevant-irrelevant label pairs (Elisseeff and Weston 2002), or relevant-relevant label pairs with different importance degrees (Xu, Li, and Zhou 2019), or output coding margins (Liu and Tsang 2015; Liu et al. 2019), etc.

It is worth noting that we adopt the same strategy in (Zhang and Yeung 2014; Liu et al. 2016; Ma and Chen 2019) by employing the regularization term $\operatorname{tr}\left(\mathbf{W C}^{-1} \mathbf{W}^{\top}\right)$ to help induce a set of learners jointly. Nonetheless, $\mathrm{M}^{3} \mathrm{MDC}$ differs from those related works which aims to solve the 
MDC problem based on maximum margin formulation. Furthermore, the empirical loss utilized by $\mathrm{M}^{3} \mathrm{MDC}$ follows from the one-vs-one decomposition w.r.t. each class space.

\section{Experiments}

\section{Experimental Setup}

Benchmark data sets. A total of ten benchmark data sets are employed for performance evaluation. Table 2 summarizes the characteristics of all MDC data sets, including number of examples (\#Exam.), number of class spaces (\#Dim.), number of class labels per class space (\#Labels/Dim.), ${ }^{2}$ and number of features (\#Features).

Comparing approaches. The performance of $\mathrm{M}^{3} \mathrm{MDC}$ is compared with four well-established MDC approaches (Read, Bielza, and Larrañaga 2014) including Binary Relevance (BR), Ensembles of Classifier Chains (ECC), Ensembles of Class Powerset (ECP), and Ensembles of Super Class classifiers (ESC). BR solves MDC problem by training a number of independent multi-class classifiers, one per class space, while ECC, ECP, ESC model dependencies among class spaces by specifying a chaining order over class spaces, conducting powerset transformation, and grouping the MDC class variables into super-classes respectively.

For ensemble approaches ECC, ECP and ESC, a random cut of $67 \%$ examples from the original MDC training set is used to generate the base MDC model and the number of base classifiers is set to be 10. Furthermore, predictions of base MDC models are combined via majority voting. Support vector machine (SVM) is used to instantiate BR, ECC, ECP, ESC as base classifier. Specifically, LIBSVM (Chang and Lin 2011) with linear kernel is used. ${ }^{3}$ As shown in Table 1, the two regularization parameters for $\mathrm{M}^{3} \mathrm{MDC}$ are set to be $\lambda_{1}=0.1, \lambda_{2}=0.001$ respectively.

Evaluation metrics. In this paper, a total of three metrics, i.e., Hamming Score, Exact Match and Sub-Exact Match, are utilized to measure the generalization abilities of MDC approaches. Specifically, let $\mathcal{S}=\left\{\left(\boldsymbol{x}_{i}, \boldsymbol{y}_{i}\right) \mid 1 \leq i \leq p\right\}$ denote the test set, where $\boldsymbol{y}_{i}=\left[y_{i 1}, y_{i 2}, \ldots, y_{i q}\right]^{\top}$ is the ground-truth class vector associated with $\boldsymbol{x}_{i}$. To evaluate the performance of the MDC predictive function $f$, let $\hat{\boldsymbol{y}}_{i}=$ $f\left(\boldsymbol{x}_{i}\right)=\left[\hat{y}_{i 1}, \hat{y}_{i 2}, \ldots, \hat{y}_{i q}\right]^{\top}$ denote the predicted class vector of $\boldsymbol{x}_{i}$, and then we can get the number of class spaces which $f$ predicts correctly, i.e., $r^{(i)}=\sum_{j=1}^{q} \llbracket y_{i j}=\hat{y}_{i j} \rrbracket$. Here, the predicate $\llbracket \pi \rrbracket$ returns 1 if $\pi$ holds and 0 otherwise. Concrete metric definitions can be given as follows:

- Hamming Score:

$$
\operatorname{HScore}_{\mathcal{S}}(f)=\frac{1}{p} \sum_{i=1}^{p} \frac{1}{q} \cdot r^{(i)}
$$

\footnotetext{
${ }^{2}$ If all class spaces have the same number of class labels, then only this number is recorded; Otherwise, the number of class labels in each class space is recorded in turn.

${ }^{3}$ Due to the margin-based nature of $\mathrm{M}^{3} \mathrm{MDC}$, we employ LIB$\mathrm{SVM}$ as the base classifier for fair comparison between $\mathrm{M}^{3} \mathrm{MDC}$ and the comparing approaches.
}

Table 2: Characteristics of the experimental data sets.

\begin{tabular}{lcccc}
\hline \hline Data Set & \#Exam. & \#Dim. & \#Labels/Dim. & \#Features $^{\dagger}$ \\
\hline Edm & 154 & 2 & 3 & $16 n$ \\
Flare1 & 323 & 3 & $3,4,2$ & $10 x$ \\
Cal500 & 502 & 10 & 2 & $68 n$ \\
Music & 591 & 6 & 2 & $71 n$ \\
Song & 785 & 3 & 3 & $98 n$ \\
WQplants & 1060 & 7 & 4 & $16 n$ \\
WQanimals & 1060 & 7 & 4 & $16 n$ \\
WaterQuality & 1060 & 14 & 4 & $16 n$ \\
Yeast & 2417 & 14 & 2 & $103 n$ \\
Voice & 3136 & 2 & 4,2 & $19 n$ \\
\hline \hline
\end{tabular}

${ }^{\dagger} n$ and $x$ denote numeric and nominal features respectively.

- Exact Match:

$$
\operatorname{EMatch}_{\mathcal{S}}(f)=\frac{1}{p} \sum_{i=1}^{p} \llbracket r^{(i)}=q \rrbracket
$$

- Sub-Exact Match:

$$
\operatorname{SEMatch}_{\mathcal{S}}(f)=\frac{1}{p} \sum_{i=1}^{p} \llbracket r^{(i)} \geq q-1 \rrbracket
$$

In a nutshell, Hamming Score is the average accuracy over all class spaces, while Exact Match is the accuracy when considering all class spaces as a single one by conducting powerset transformation. Sub-Exact Match is a relaxed version of Exact Match where at most one incorrect prediction can be made over all class spaces for each test example. For all three metrics, the larger the values the better the performance. Ten-fold cross-validation is performed on the benchmark data sets, where the mean metric value as well as standard deviation are recorded for each comparing approach.

\section{Experimental Results}

Table 3 reports the detailed experimental results of five comparing approaches in terms of each evaluation metric, where the best performance among all comparing approaches is shown in boldface. Moreover, Wilcoxon signed-ranks test (Demšar 2006) is used as the statistical test to show whether $\mathrm{M}^{3}$ MDC performs significantly better than BR, ECC, ECP, ESC respectively. Table 4 summarizes the statistical test results and the $p$-values for the corresponding tests are also shown in the brackets. Here, the significance level is set to be 0.05 .

Based on the reported experimental results, the following observations can be made:

- Across all the 30 cases (10 data sets $\times 3$ evaluation metrics), $\mathrm{M}^{3} \mathrm{MDC}$ ranks first in 21 cases, ranks second in 3 cases, and never ranks last.

- In terms of Hamming Score, $\mathrm{M}^{3} \mathrm{MDC}$ is statistically better than BR, ECC, ECP, ESC.

- ECP can be regarded as an approach which works by maximizing Exact Match via class powerset transformation. It is worth noting that $\mathrm{M}^{3} \mathrm{MDC}$ still ranks first in 5 out of 10 cases in term of this metric and can achieve comparable performance against ECP. 
Table 3: Predictive performance of each comparing approach (mean \pm std. deviation) on experimental data sets. Moreover, the best performance among all comparing approaches is shown in boldface.

(a) Hamming Score

\begin{tabular}{|c|c|c|c|c|c|c|c|c|c|c|}
\hline Data Set & Edm & Flare1 & Cal500 & Music & Song & WQpla. & WQani. & WQ & Yeast & Voice \\
\hline $\mathrm{M}^{3} \mathrm{MDC}$ & $.728 \pm .083$ & $.923 \pm .033$ & $.630 \pm .010$ & $.811 \pm .022$ & $.795 \pm .029$ & $.660 \pm .013$ & $.632 \pm .014$ & $.647 \pm .012$ & $.802 \pm .006$ & $.971 \pm .009$ \\
\hline BR & $.689 \pm .070$ & $.922 \pm .034$ & $.628 \pm .011$ & $.808 \pm$ & $.793 \pm$. & $.657=$ & .630 & 13 & 6 & 007 \\
\hline ECC & $.695 \pm .065$ & $.922 \pm .034$ & $.625 \pm .015$ & $.814 \pm .025$ & $.790 \pm .024$ & $.654 \pm .016$ & $.630 \pm .014$ & $.643 \pm .013$ & $.797 \pm .007$ & $.961 \pm .008$ \\
\hline ECP & $.721 \pm .082$ & $.921 \pm .036$ & $.616 \pm .015$ & $.799 \pm .032$ & $.786 \pm .029$ & $.647 \pm .015$ & $.629 \pm .013$ & $.628 \pm .015$ & $.795 \pm .007$ & $.955 \pm .013$ \\
\hline ESC & $.701 \pm .079$ & $.923 \pm .033$ & $.616 \pm .019$ & $.809 \pm .022$ & $.790 \pm .030$ & $.651 \pm .016$ & $.630 \pm .014$ & $.641 \pm .013$ & $.800 \pm .006$ & $.961 \pm .008$ \\
\hline
\end{tabular}

(b) Exact Match

\begin{tabular}{|c|c|c|c|c|c|c|c|c|c|c|}
\hline Data Set & $\overline{E d m}$ & Flare1 & Cal500 & Music & Song & WQpla. & WQani. & WQ & Yeast & Voice \\
\hline $\mathrm{M}^{3} \mathrm{MDC}$ & $.501 \pm .139$ & $.821 \pm .073$ & $.016 \pm .016$ & $.281 \pm .074$ & $.488 \pm .065$ & $.102 \pm .035$ & $.059 \pm .022$ & $.008 \pm .008$ & $.157 \pm .018$ & $.942 \pm .017$ \\
\hline BR & $.442 \pm .125$ & $.821 \pm .073$ & $.016 \pm .016$ & $.272 \pm .075$ & $.479 \pm .059$ & $.097 \pm .033$ & $.058 \pm .022$ & $.007 \pm .008$ & $.151 \pm .017$ & $.929 \pm .014$ \\
\hline ECC & $.454 \pm .123$ & $.817 \pm .078$ & $.020 \pm .016$ & $.346 \pm .079$ & $.481 \pm .057$ & $.093 \pm .037$ & $.061 \pm .023$ & $.006 \pm .008$ & $.207 \pm .014$ & $.923 \pm .016$ \\
\hline ECP & $.559 \pm .136$ & $.817 \pm .078$ & $.026 \pm .028$ & $.343 \pm .076$ & $.484 \pm .054$ & $.093 \pm .028$ & $.065 \pm .018$ & $.001 \pm .003$ & $.252 \pm .012$ & $.912 \pm .025$ \\
\hline ESC & $.513 \pm .122$ & $.821 \pm .073$ & $.014 \pm .013$ & $.330 \pm .069$ & $.480 \pm .067$ & $.094 \pm .038$ & $.062 \pm .021$ & $.006 \pm .008$ & $.236 \pm .019$ & $.924 \pm .016$ \\
\hline
\end{tabular}

(c) Sub-Exact Match

\begin{tabular}{|c|c|c|c|c|c|c|c|c|c|c|}
\hline Data Set & Edm & Flare1 & Cal500 & Music & Song & WQpla. & WQani. & WQ & Yeast & Voice \\
\hline $\mathrm{M}^{3} \mathrm{MDC}$ & $.955 \pm .053$ & $.951 \pm .036$ & $.082 \pm .046$ & $.687 \pm .067$ & $.901 \pm .042$ & $.287 \pm .051$ & $.237 \pm .028$ & $.051 \pm .025$ & $.273 \pm .028$ & $.999 \pm .001$ \\
\hline BR & $.935 \pm .061$ & $.947 \pm .039$ & $.074 \pm .037$ & $.674 \pm .067$ & $.903 \pm .033$ & $.287 \pm .055$ & $.229 \pm .034$ & $.051 \pm .024$ & $.269=$ & $.999 \pm .002$ \\
\hline ECC & $.935 \pm .069$ & $.951 \pm .036$ & $.080 \pm .031$ & $.676 \pm .064$ & $.891 \pm .036$ & $.283 \pm .049$ & $.229 \pm .032$ & $.050 \pm .023$ & $.288 \pm .023$ & $.998 \pm .002$ \\
\hline ECP & $.883 \pm .074$ & $.947 \pm .039$ & $.078 \pm .036$ & $.640 \pm .064$ & $.878 \pm .040$ & $.281 \pm .049$ & $.230 \pm .032$ & $.035 \pm .018$ & $.304 \pm .020$ & $.998 \pm .003$ \\
\hline ESC & $.890 \pm .076$ & $.951 \pm .036$ & $.086 \pm .038$ & $.669 \pm .062$ & $.893 \pm .038$ & $.284 \pm .050$ & $.232 \pm .033$ & $.046 \pm .022$ & $.309 \pm .028$ & $.998 \pm .002$ \\
\hline
\end{tabular}

Table 4: Wilcoxon signed-ranks test for $\mathrm{M}^{3} \mathrm{MDC}$ against BR,ECC,ECP,ESC in terms of each evaluation metric (significance level $\alpha=0.05 ; p$-values shown in the brackets).

\begin{tabular}{|c|c|c|c|c|}
\hline Evaluation Metric & $\mathrm{M}^{3} \mathrm{MDC}$ vs BR & $\mathrm{M}^{3} \mathrm{MDC}$ vs ECC & $\mathrm{M}^{3} \mathrm{MDC}$ vs ECP & $\mathrm{M}^{3} \mathrm{MDC}$ vs ESC \\
\hline Hamming Score & win $[1.95 \mathrm{e}-3]$ & win $[9.77 \mathrm{e}-3]$ & win $[1.95 \mathrm{e}-3]$ & win $[3.91 \mathrm{e}-3]$ \\
\hline Exact Match & win [7.81e-3] & tie $[7.70 \mathrm{e}-1]$ & tie $[4.32 \mathrm{e}-1]$ & tie $[7.54 \mathrm{e}-1]$ \\
\hline Sub-Exact Match & win $[2.34 \mathrm{e}-2]$ & tie $[9.77 \mathrm{e}-2]$ & win [4.88e-2] & tie $[1.95 \mathrm{e}-1]$ \\
\hline
\end{tabular}

- It is impressive to notice that $\mathrm{M}^{3} \mathrm{MDC}$ is statistically better than BR in terms of all evaluation metrics, which clearly validates the effectiveness of $\mathrm{M}^{3} \mathrm{MDC}$ in modeling relationships among class spaces.

\section{Further Analysis}

Sensitivity analysis. As shown in Eq.(2), $\lambda_{1}, \lambda_{2}$ are used to make a tradeoff among empirical risk, structural risk and relationship regularizer. Figure 1 shows how the performance of $\mathrm{M}^{3}$ MDC changes w.r.t. $\lambda_{1}, \lambda_{2}$ on data sets Music and Song respectively. Similar results can be obtained on other data sets which are not reported here due to page limit. In terms of each evaluation metric, $\mathrm{M}^{3} \mathrm{MDC}$ can achieve relatively better performance when $\lambda_{1}=0.1$ and $\lambda_{2} \leq 1$. In this paper, we fix $\lambda_{1}=0.1, \lambda_{2}=0.001$ respectively, which are also the recommended default parameter settings for ease of use.

Correlation analysis. By normalizing matrix $\mathbf{C}$ in Eq.(2) with its diagonal elements, we can get correlation matrix $\mathbf{R}$ which represents the relationships among all pairs of class labels, i.e., $R_{i j}=\frac{C_{i j}}{\sqrt{C_{i i} \times C_{j j}}}$, where $R_{i j}\left(C_{i j}\right)$ denotes ele- ment in $i$ th row and $j$ th column of $\mathbf{R}(\mathbf{C})$. We depict the correlation matrix $\mathbf{R}$ on data sets Song, WaterQuality and Yeast in Figure 2. Here, +1 indicates absolutely positive correlation (i.e., red color) while -1 indicates absolutely negative correlation (i.e., blue color). As shown in Figure 2, there are indeed some red or blue squares (excluding diagonal ones), which indicate that dependencies among classes do exist. However, there are many squares in green which indicate independencies between classes. These observations show that class dependencies should indeed be taken into account but with great care when designing MDC approaches. $\mathrm{M}^{3} \mathrm{MDC}$ can model class dependencies automatically as long as dependencies exist which is a desirable property when inducing predictive models.

Convergence analysis. The optimization problem in Eq.(3) is solved in an alternating way. Although the objective function is jointly convex, here we also analyze its convergent characteristics. Specifically, Frobenius norm of the difference between each pair of $\mathbf{W}$ s in two adjacent iterations is recorded. Figure 3 illustrates how the Frobenius norm changes as the number of iterations increases on data sets Song and Voice. It is observed that the difference has 


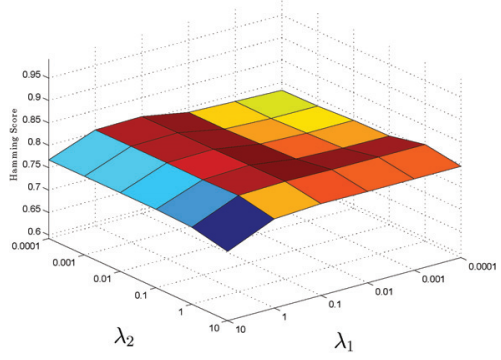

(a) Hamming Score (Music)

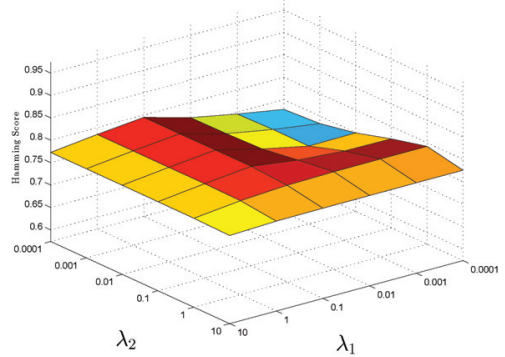

(d) Hamming Score (Song)

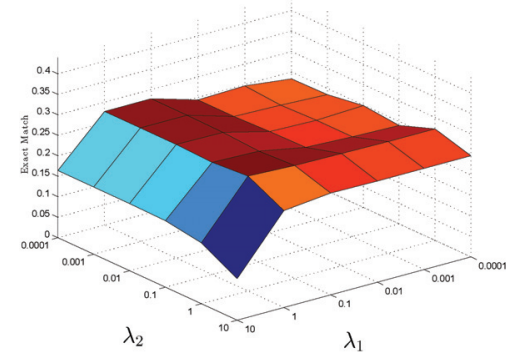

(b) Exact Match (Music)

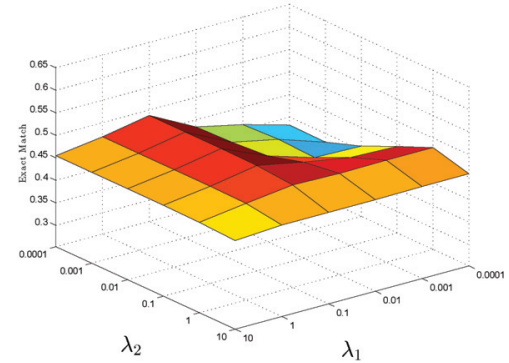

(e) Exact Match (Song)

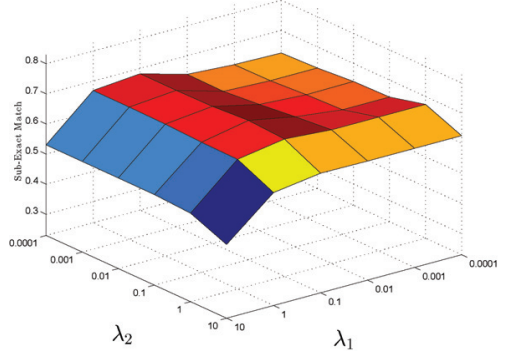

(c) Sub-Exact Match (Music)

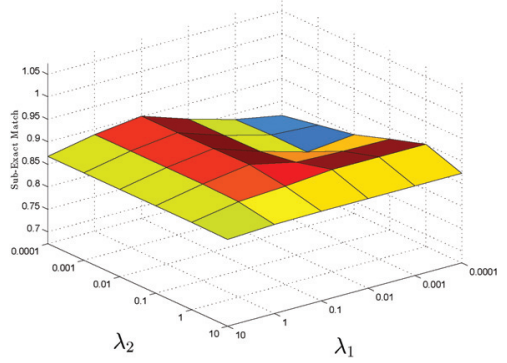

(f) Sub-Exact Match (Song)

Figure 1: Performance of $\mathrm{M}^{3} \mathrm{MDC}$ changes as $\lambda_{1}, \lambda_{2}$ range in $\{10,1,0.1,0.01,0.001,0.0001\}$

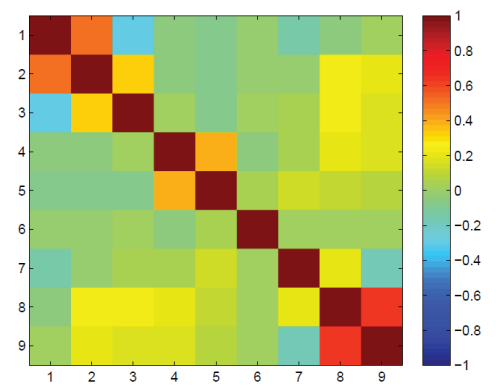

(a) Song

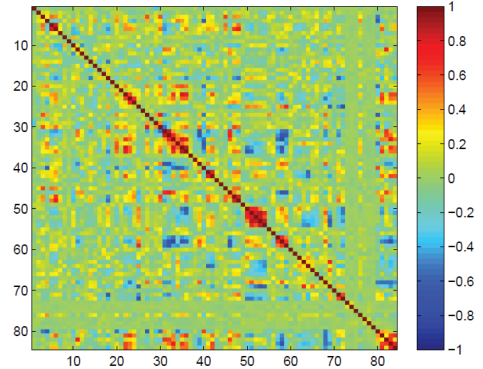

(b) WaterQuality

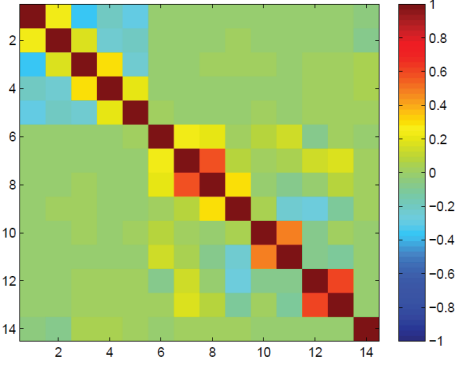

(c) Yeast

Figure 2: Correlation matrix on data sets Song, WaterQuality, and Yeast

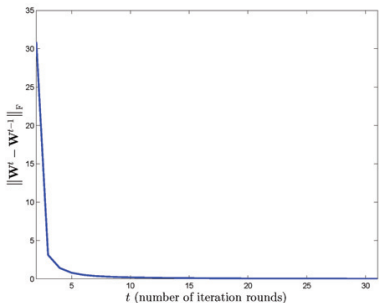

(a) Song

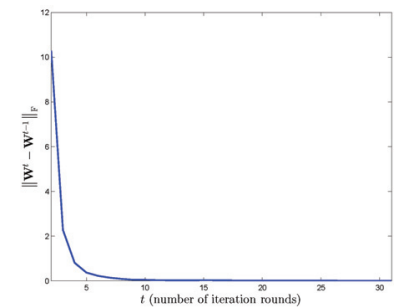

(b) Voice
Figure 3: Convergence curves on data sets Song and Voice.

been very small when the number of iterations reaches 5 , which means $\mathrm{M}^{3} \mathrm{MDC}$ usually converges very quickly.

\section{Conclusion}

In this paper, the problem of margin-based multidimensional classification is investigated. Specifically, a novel approach named $\mathrm{M}^{3} \mathrm{MDC}$ is proposed which considers the margin over MDC examples via OvO decomposition and models the dependencies among class spaces with covariance regularization. The resulting convex formulation is solved via alternating optimization admitting QP or closedform solution in either alternating step. Experimental studies on benchmark data sets clearly validate the effectiveness of the derived $\mathrm{M}^{3} \mathrm{MDC}$ approach.

\section{Acknowledgments}

The authors wish to thank the anonymous reviewers for their helpful comments and suggestions. This work was supported by the National Key R\&D Program of China (2018YFB1004300), the National Science Foundation of 
China (61573104), and partially supported by the Collaborative Innovation Center of Novel Software Technology and Industrialization.

\section{References}

Arias, J.; Gamez, J. A.; Nielsen, T. D.; and Puerta, J. M. 2016. A scalable pairwise class interaction framework for multidimensional classification. International Journal of Approximate Reasoning 68:194-210.

Batal, I.; Hong, C.; and Hauskrecht, M. 2013. An efficient probabilistic framework for multi-dimensional classification. In Proceedings of the 22nd ACM International Conference on Information \& Knowledge Management, 24172422.

Benjumeda, M.; Bielza, C.; and Larrañaga, P. 2018. Tractability of most probable explanations in multidimensional bayesian network classifiers. International Journal of Approximate Reasoning 93:74-87.

Bielza, C.; Li, G.; and Larrañaga, P. 2011. Multidimensional classification with bayesian networks. International Journal of Approximate Reasoning 52(6):705-727.

Bolt, J. H., and van der Gaag, L. C. 2017. Balanced sensitivity functions for tuning multi-dimensional bayesian network classifiers. International Journal of Approximate Reasoning 80:361-376.

Borchani, H.; Bielza, C.; Toro, C.; and Larrañaga, P. 2013. Predicting human immunodeficiency virus inhibitors using multi-dimensional bayesian network classifiers. Artificial Intelligence in Medicine 57(3):219-229.

Chang, C.-C., and Lin, C.-J. 2011. LIBSVM: A library for support vector machines. ACM Transactions on Intelligent Systems and Technology 2(3):Article 27. Software available at http://www.csie.ntu.edu.tw/ cjlin/libsvm.

Cortes, C., and Vapnik, V. 1995. Support-vector networks. Machine Learning 20(3):273-297.

Demšar, J. 2006. Statistical comparisons of classifiers over multiple data sets. Journal of Machine Learning Research 7(Jan):1-30.

Elisseeff, A., and Weston, J. 2002. A kernel method for multi-labelled classification. In Advances in Neural Information Processing Systems, 681-687.

Gibaja, E., and Ventura, S. 2015. A tutorial on multilabel learning. ACM Computing Surveys 47(3):Article 52.

Gil-Begue, S.; Larrañaga, P.; and Bielza, C. 2018. Multidimensional bayesian network classifier trees. In Lecture Notes in Computer Science 11314. Switzerland: Springer. 354-363.

Jia, B.-B., and Zhang, M.-L. 2019. Multi-dimensional classification via kNN feature augmentation. In Proceedings of the 33rd AAAI Conference on Artificial Intelligence, 39753982.

Liu, W., and Tsang, I. W. 2015. Large margin metric learning for multi-label prediction. In Proceedings of the 29th AAAI Conference on Artificial Intelligence, 2800-2806.
Liu, M.; Zhang, D.; Chen, S.; and Xue, H. 2016. Joint binary classifier learning for ecoc-based multi-class classification. IEEE Transactions on Pattern Analysis and Machine Intelligence 38(11):2335-2341.

Liu, W.; Xu, D.; Tsang, I. W.; and Zhang, W. 2019. Metric learning for multi-output tasks. IEEE Transactions on Pattern Analysis and Machine Intelligence 41(2):408-422.

Ma, Z., and Chen, S. 2018. Multi-dimensional classification via a metric approach. Neurocomputing 275:1121-1131.

Ma, Z., and Chen, S. 2019. A convex formulation for multiple ordinal output classification. Pattern Recognition 86:7384.

Read, J.; Bielza, C.; and Larrañaga, P. 2014. Multidimensional classification with super-classes. IEEE Transactions on Knowledge and Data Engineering 26(7):17201733.

Read, J.; Martino, L.; and Luengo, D. 2014. Efficient monte carlo methods for multi-dimensional learning with classifier chains. Pattern Recognition 47(3):1535-1546.

Rodríguez, J. D.; Pérez, A.; Arteta, D.; Tejedor, D.; and Lozano, J. A. 2012. Using multidimensional bayesian network classifiers to assist the treatment of multiple sclerosis. IEEE Transactions on Systems, Man, and Cybernetics Part C: Applications and Reviews 42(6):1705-1715.

Sagarna, R.; Mendiburu, A.; Inza, I.; and Lozano, J. A. 2014. Assisting in search heuristics selection through multidimensional supervised classification: A case study on software testing. Information Sciences 258:122-139.

Serafino, F.; Pio, G.; Ceci, M.; and Malerba, D. 2015. Hierarchical multidimensional classification of web documents with multiwebclass. In Lecture Notes in Computer Science 9356. Berlin: Springer. 236-250.

Xu, M.; Li, Y.-F.; and Zhou, Z.-H. 2019. Robust multi-label learning with PRO loss. IEEE Transactions on Knowledge and Data Engineering, in press.

Zaragoza, J. H.; Sucar, L. E.; Morales, E. F.; Bielza, C.; and Larrañaga, P. 2011. Bayesian chain classifiers for multidimensional classification. In Proceedings of the 22nd International Joint Conference on Artificial Intelligence, volume 11, 2192-2197.

Zhang, Y., and Yeung, D.-Y. 2014. A regularization approach to learning task relationships in multi-task learning. ACM Transactions on Knowledge Discovery from Data 8(3):Article 12.

Zhang, M.-L., and Zhou, Z.-H. 2014. A review on multilabel learning algorithms. IEEE Transactions on Knowledge and Data Engineering 26(8):1819-1837.

Zhu, M.; Liu, S.; and Jiang, J. 2016. A hybrid method for learning multi-dimensional bayesian network classifiers based on an optimization model. Applied Intelligence 44(1):123-148. 